\title{
Histone deacetylase inhibitors induce in human hepatoma HepG2 cells acetylation of p53 and histones in correlation with apoptotic effects
}

\author{
D. CARLISI, B. VASSALLO, M. LAURICELLA, S. EMANUELE, A. D'ANNEO, \\ E. DI LEONARDO, P. DI FAZIO, R. VENTO and G. TESORIERE
}

Dipartimento di Scienze Biochimiche, Università di Palermo, Policlinico, Palermo, Italy

Received August 9, 2007; Accepted September 27, 2007

\begin{abstract}
This report shows that histone deacetylase inhibitors (HDACIs) induced apoptosis in human hepatoma HepG2 cells in a dose- and time-dependent manner. Trichostatin A (TSA), ITF2357 and suberoylanilide hydroxamic acid (SAHA), which were very effective agents, caused apoptotic effects after a lag phase of 12-16 h. In order to elucidate the mechanism of HDACIs action in HepG2 cells we have studied the effects of TSA, ITF2357 and SAHA on acetylation of p53 and histones $\mathrm{H} 2 \mathrm{~A}, \mathrm{H} 2 \mathrm{~B}, \mathrm{H} 3$ and $\mathrm{H} 4$. It was observed that HDACIs rapidly induced acetylation of these proteins, being the effects clearly visible already at 30 min of treatment at the same doses which caused apoptosis. Analysis of the immunocomplexes, obtained from nuclear extracts using an antibody against p53, revealed the presence of acetylated p53 together with acetylated forms of histones and histone acetyltransferases p300 and PCAF. Experiments performed using pifithrin- $\alpha$, a reversible inhibitor of p53, showed a correlation between acetylation of p53 and induction of apoptosis. In addition treatment with siRNA against p53 indicated that p53 is involved in the acetylation of histones. In conclusion, this report suggests that complexes constituted by acetylated p53, acetylated histones and coactivators can play a central role in HDACI-induced apoptosis in HepG2 cells.
\end{abstract}

\section{Introduction}

The acetylation status of histones is regulated in eukaryotes by two kinds of enzymes, histone acetyltransferases (HATs) and histone deacetylases (HDACs), which are responsible for acetylation and deacetylation of lysines residues in $\mathrm{N}$-terminal tails of histones (1).

Correspondence to: Professor Giovanni Tesoriere, Dipartimento di Scienze Biochimiche, Policlinico, Via del Vespro 129, I-90127 Palermo, Italy

E-mail: gtesor@unipa.it

Key words: histone deacetylase inhibitors, acetylation, p53, histones, apoptosis, hepatoma cells
Acetylation status of histones plays a crucial role in the control of gene expression. Suppression of positive charges in the histone tails induces relaxation of chromatin structure favouring DNA accessibility to transcriptional factors with increased transcription of target genes involved in apoptosis (2), while deacetylation reduces DNA availability (3). Thus acetylation, together with phosphorylation and methylation of N-terminal tail of histones, are involved in regulating funda-mental processes, such as proliferation and cell death $(4,5)$. Sodium butyrate, which belongs together with valproic acid to the class of short chain fatty acids, was the first HDAC inhibitor (HDACI) to be identified (1). However, butyrates are less potent than other HDACIs (6). Recently the class of hydroxamic acid-derived compounds has attracted the interest of researchers. This class includes Trichostatin A (TSA), derived from metabolic products of streptomycetes, suberoylanilide hydroxamic acid (SAHA) and ITF2357 (7).

The mechanism of action of hydroxamic acid group consists of the interaction with catalytic site of HDAC blocking the substrate approach to zinc ion present in this catalytic structure (8). The consequent hyperacetylation of histones produces changes in chromatin structure promoting binding of transcriptional factors to DNA $(1,9)$. Thus HDACIs may cause transcriptional activation of a set of genes inducing growth arrest and apoptosis with inhibition of tumor growth. In particular SAHA has been shown to provoke marked apoptotic effects on a number of tumor cell types (10-13), whereas normal cells appear to be relatively resistant. In addition, SAHA showed a high therapeutic potential for different forms of tumors at doses that were well tolerated by patients $(14,15)$. We have recently demonstrated (16) that SAHA induces apoptosis in human hepatoma HepG2 cells by activating both the extrinsic and the intrinsic pathways of apoptosis. In particular SAHA increased in HepG2 cells the level of both FasL and Fas receptor and that of the apoptotic members of Bcl-2 family. In addition the drug caused loss of mitochondrial transmembrane potential and release of cytochrome $\mathrm{c}$ from mitochondria and finally activated both caspases 8 and 3. Although it appears clear that SAHA produces many different effects, it is possible that all these modifications can be a consequence of some initial fundamental events. 
In this report we propose that these initial effects can be represented by acetylation of p53 and histones. Moreover, we suggest that the production of nuclear complexes containing acetylated forms of p53 and histones together with histone acetyltransferases can be correlated with the activation of a set of apoptotic genes.

\section{Materials and methods}

Cell cultures and reagents. Human hepatoma HepG2 cells were grown as monolayers in RPMI-1640 medium supplemented with $10 \%(\mathrm{v} / \mathrm{v})$ heat-inactivated fetal calf serum (FCS), $1.0 \mathrm{mM}$ sodium pyruvate and $2.0 \mathrm{mM}$ glutamine and maintained at $37^{\circ} \mathrm{C}$ in a humidified atmosphere containing $5 \% \mathrm{CO}_{2}$. For the experiments, cells were seeded on 6-well plates $\left(2.5 \times 10^{5}\right.$ cells $)$ or $100-\mathrm{mm}$ tissue culture dishes $\left(1 \times 10^{6}\right.$ cells). After plating, cells were allowed to adhere overnight and then treated with chemicals or vehicle (control cells). All chemicals, except when stated otherwise, were obtained from Sigma (St. Louis, MO). Suberoylanilide hydroxamic acid (SAHA) and ITF2357 were a kind gift of Italfarmaco S.p.a. (Milan, Italy). Stock solutions of both SAHA and ITF2357 were prepared in dimethyl sulfoxide (DMSO) and diluited to their final concentrations in the culture medium. The final concentration of DMSO never exceeded $0.04 \%$, which is a concentration that had no discernible effect compared to the control HepG2 cells.

Assessment of apoptosis and cell cycle analysis. To analyze cell cycle distribution by flow cytometric analysis, after treatment with the compounds, cells were harvested by trypsinization and resuspended in a hypotonic solution containing $50 \mu \mathrm{g} / \mathrm{ml}$ propidium iodide, $0.1 \%$ sodium citrate, $0.01 \%$ Nonidet P-40 and $10 \mu \mathrm{g} / \mathrm{ml}$ RNAse A and incubated overnight at $4^{\circ} \mathrm{C}$. The cell cycle phase distribution was evaluated by an Epics $\mathrm{XL}$ flow cytometer (Beckman Coulter). The percentage of cells giving fluorescence in the hypodiploid sub-G0/G1 peak of cell cycle was taken as a measure of apoptosis. Data were analysed using Expo32 software.

Western blot analysis. The levels of apoptotic factors were analysed by means of Western blot analysis using specific antibodies. Cell lysates were prepared as reported $(17,18)$. Protein concentration was determined as described by Lowry et al (19). Equal amounts of proteins (40 $\mu \mathrm{g} /$ lane) were resolved by SDS-PAGE and electroblotted to nitrocellulose membranes. The filters were then probed using the following specific antibodies: mouse monoclonal anti-p53 (DO1), mouse monoclonal anti-MDM2, rabbit polyclonal anti-p21, goat polyclonal anti-Ac-histone $\mathrm{H} 2 \mathrm{~B}$, goat polyclonal antiAc-histone $\mathrm{H} 3$, rabbit polyclonal anti-Ac-histone $\mathrm{H} 4$, goat polyclonal anti-PCAF, rabbit polyclonal anti-p300 and mouse monoclonal anti-actin, obtained from Santa Cruz Biotechnology (St. Cruz, CA); rabbit polyclonal anti-Ac-histone $\mathrm{H} 2 \mathrm{~A}$, rabbit antiserum acetyl-lysine, rabbit antiserum AcLys320 p53 and rabbit antiserum Ac-Lys373/382 p53, obtained from Upstate (Temecula, CA). Bands were visualized using ECL detection kit (Pierce). Immunoreactive bands were quantified by using Fluor-S-Max Chemidoc system and Quantity One software (Bio-Rad). For each experiment, actin blots were included to show equal protein loading for all the samples.

Immunoprecipitation analysis. HepG2 cells $\left(1 \times 10^{7}\right.$ cells $)$ were homogenized in dounce with $20 \mathrm{mM}$ HEPES, containing $250 \mathrm{mM}$ sucrose, $10 \mathrm{mM} \mathrm{KCl}, 1.5 \mathrm{mM} \mathrm{MgCl}_{2}, 1 \mathrm{mM}$ EDTA, $1 \mathrm{mM}$ EGTA, $1 \mathrm{mM}$ DTT and a mix of protease inhibitors (0.5 mM PMSF, $10 \mu \mathrm{g} / \mathrm{ml}$ leupeptin, $10 \mu \mathrm{g} / \mathrm{ml}$ aprotinin, $0.7 \mu \mathrm{g} / \mathrm{ml}$ pepstatin), $\mathrm{pH} 7.5$. Homogenates were centrifuged at $1,500 \mathrm{xg}$ for $10 \mathrm{~min}$ at $4^{\circ} \mathrm{C}$ and the pellets re-homogenized in the same buffer. After a centrifugation at $800 \mathrm{x}$ g for $10 \mathrm{~min}$, nuclei were resuspended in RIPA buffer $(50 \mathrm{mM}$ Tris- $\mathrm{HCl}$ $\mathrm{pH} 7.4$, containing $1 \% \mathrm{NP}-40,1 \mathrm{mM}$ EDTA and protease inhibitors), mixed by multiple passage through pasteur and then centrifuged at 13,000 x g for $10 \mathrm{~min}$ (step 1). Afterwards, pellets were resuspended in RIPA buffer, and re-extracted as described in step 1 (step 2). The supernatants from both steps were mixed, precleared with $5 \mu 1$ of protein-AG agarose (Santa Cruz Biotecnology) for $30 \mathrm{~min}$ at $4^{\circ} \mathrm{C}$ and then centrifuged for $10 \mathrm{~min}$ at $13,000 \mathrm{x} \mathrm{g}$.

For immunoprecipitation procedures equal amounts of proteins $(400 \mu \mathrm{g})$ were incubated with $0.4 \mu \mathrm{g}$ of mouse monoclonal p53 (DO1) antibody or rabbit antiserum acetyllysine antibody overnight at $4^{\circ} \mathrm{C}$ in a total volume of $200 \mu 1$ of RIPA buffer with protease inhibitors. Then protein A/Gagarose was added and the incubation was protracted overnight at $4^{\circ} \mathrm{C}$. The immunocomplexes were centrifuged at $10,000 \mathrm{x} \mathrm{g}$ for $5 \mathrm{~min}$, washed three times in PBS, and dissolved into $4 \mathrm{X}$ SDS loading buffer at $90^{\circ} \mathrm{C}$ for $10 \mathrm{~min}$. The proteins were analyzed by Western blot with different antibodies.

siRNA transfection. p53 siRNA and scrambled sequence were obtained from Santa Cruz Biotechnology. Cells were plated into 6-well plates (35-mm) and cultured in RPMI-1640 medium antibiotic-free for $24 \mathrm{~h}$ before transfection to reach $\sim 50 \%$ confluence. For each transfection, $8 \mu 1$ of lipofectin was diluited in $100 \mu 1$ siRNA Transfection Medium (Santa Cruz Biotechnology) and incubated for $15 \mathrm{~min}$ at room temperature; in another tube 80 pmoles of p53 siRNA or scrambled sequence was mixed with $100 \mu 1$ siRNA Transfection Medium. These two solutions were combined, gently mixed and incubated for $30 \mathrm{~min}$ at room temperature to allow the production of lipofectin siRNA complex. Cells were then washed with PBS twice and incubated with siRNA lipofectin complex in siRNA Transfection Medium without FCS for $24 \mathrm{~h}$ at $37^{\circ} \mathrm{C}$. Afterwards RPMI-1640 medium containing $10 \%$ FCS was added. The cells were harvested at the indicated times for Western blot or cytofluorimetric analysis.

\section{Results}

Effects of HDACIs on apoptosis. In this study initially we ascertained by means of flow cytometric analysis the effects induced by different HDAC inhibitors on cell cycle distribution of human hepatoma HepG2 cells. The percentage of cells in sub-G0/G1 phase, corresponding to cells with subdiploid DNA, was chosen to quantify the apoptotic effect induced by HDACIs. As shown in Fig. 1, treatment with trichostatin A (TSA), ITF2357, suberoylanilide hydroxamic acid (SAHA), sodium butyrate $(\mathrm{NaB})$ or valproic acid (VPA) exerted a clear 


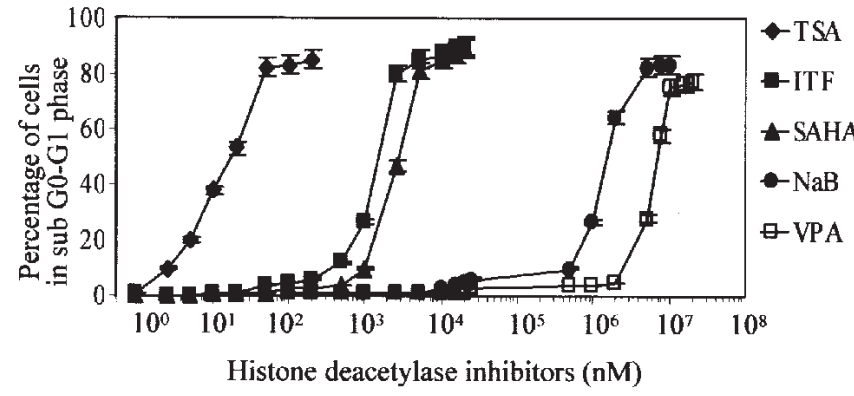

Figure 1. Dose-dependence of the apoptotic effect exerted on HepG2 cells by various inhibitors of histone deacetylases. Flow cytometric analysis of propidium iodide-stained HepG2 cells treated for $36 \mathrm{~h}$ with different doses of Trichostatin A (TSA), ITF2357, SAHA, sodium butyrate or valproic acid. The $\mathrm{x}$-axis indicates the concentrations of HDACIs on logarithmic scale; the $\mathrm{y}$-axis indicates the percentage of cells in sub-G0/G1 phase, corresponding to apoptotic cells with subdiploid DNA. The results were representative of three independent experiments $\pm \mathrm{SE}$

apoptotic effect in a dose-dependent manner, although with a different efficacy. Comparison of the doses causing apoptotic effects in a percentage of $50 \%$ of HepG2 cells (Apoptotic Index 50, $\mathrm{ApI}_{50}$ ) revealed that TSA, ITF2357 and SAHA were effective agents, showing $\mathrm{ApI}_{50}$ equal respectively to $20 \mathrm{nM}, 1.5$ and $2.5 \mu \mathrm{M}$, while a scarce effectiveness was observed for $\mathrm{NaB}$ and VPA, which showed higher values of $\mathrm{ApI}_{50}$, corresponding to 1.5 and $7.0 \mathrm{mM}$ respectively.

Experiments were also performed in order to evaluate the time-dependence of these effects. Thus, HepG2 cells were exposed for different times to TSA, SAHA or ITF2357. As shown in Fig. 2, the apoptotic effect appeared for the three compounds after a lag-phase of 12-16 h. Then, the effect increased with the time of treatment, reaching the maximum at about $36 \mathrm{~h}$.

HDACIs induced histone acetylation in HepG2 cells. We hypothesised that inhibition of HDACs can lead to histone acetylation favouring chromatin relaxation. Therefore, lysates obtained from cells treated with the histone deacetylase inhibitor SAHA were employed to ascertain the presence of acetylated forms of histones. Our results reported in Fig. 3A demonstrate that SAHA significantly increased the levels of acetyl-H2A, acetyl-H2B and acetyl-H4, while a minor effect was observed for acetyl-H3. These effects were precocious, clearly appearing at $30 \mathrm{~min}$ of exposure and increasing with the time of treatment. Fig. 3B shows the dose-dependence of the effect exerted by SAHA on acetylation of histone $\mathrm{H} 4$. Also TSA and ITF2357 induced rapid acetylation of histones in a dose- and time-dependent manner (not shown). Fig. 3C shows the relationship between the dose employed of TSA, ITF2357 and SAHA and their effects on acetylation of histone H4. For each compound the doses which caused 50\% of acetylation of histone $\mathrm{H} 4$ were coincident to those which induced apoptotic effect in a percentage of cells equal to $50 \%$ (Fig. 1).

SAHA induced acetylation of p53 and the production of complexes by interaction of p53 with acetylated histones and

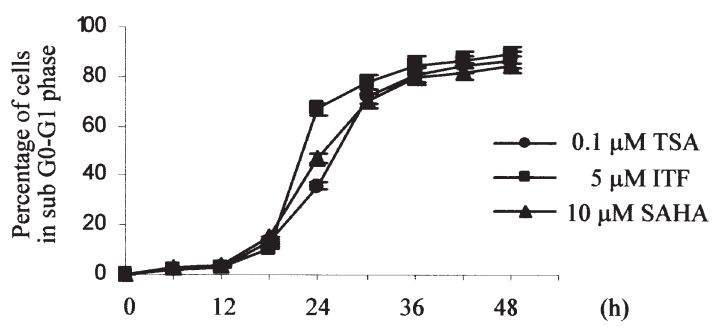

Figure 2. Time-course of the apoptotic effect exerted on HepG2 cells by TSA, ITF2357 or SAHA. Flow cytometric analysis of propidium iodide-stained HepG2 cells treated for different times with $0.1 \mu \mathrm{M}$ TSA, $5 \mu \mathrm{M}$ ITF2357 or $10 \mu \mathrm{M}$ SAHA. The percentage of cells in subdiploid region was assumed as index of apoptosis. The results were representative of three independent experiments $\pm \mathrm{SE}$
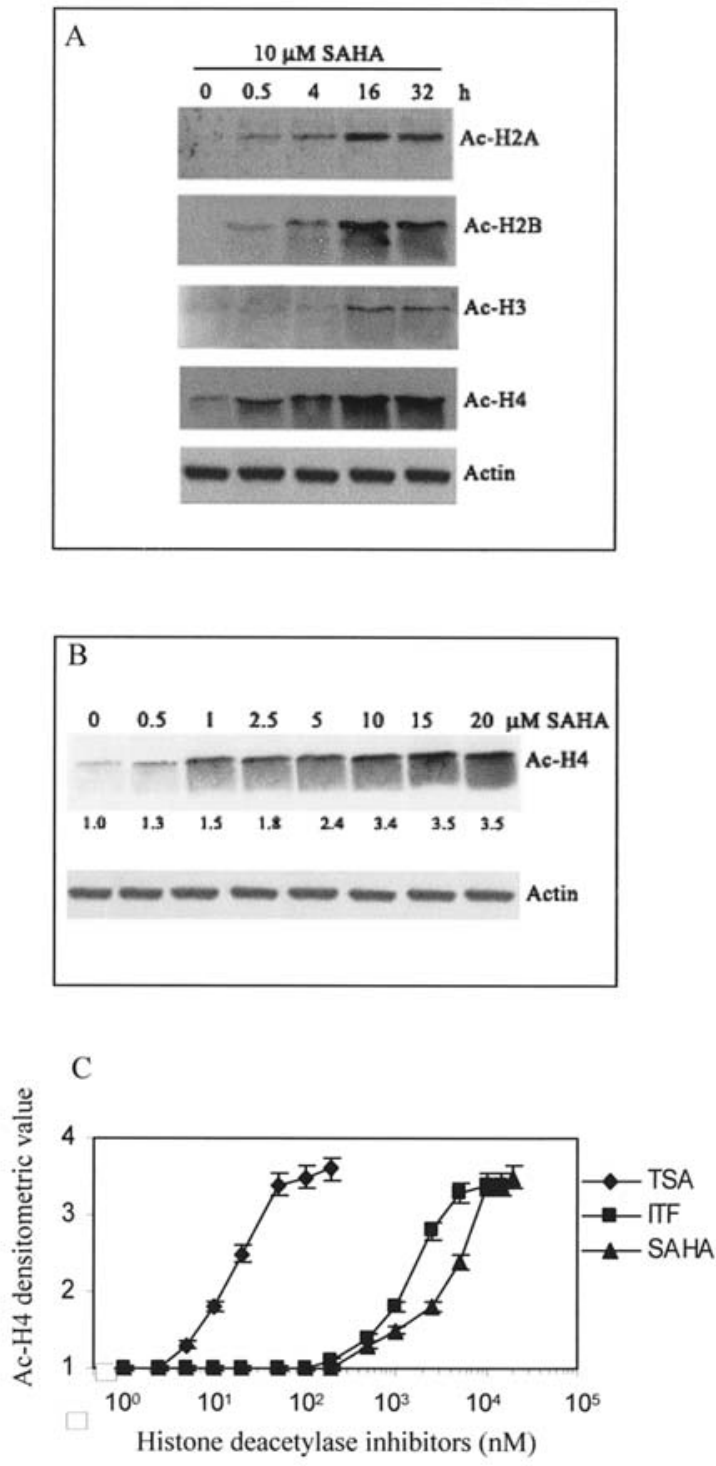

Figure 3. HDACIs induced rapid acetylation of histones. (A) HepG2 cells were treated with $10 \mu \mathrm{M}$ SAHA for various times or (B) with various doses of SAHA for $24 \mathrm{~h}$. Cell lysates were prepared and analysed in duplicate samples by Western blot using antibodies which recognize acetyl-H2A, acetyl-H2B, acetyl-H3 or acetyl-H4. (C) HepG2 cells were treated for $24 \mathrm{~h}$ with various doses of TSA, ITF2357 and SAHA. At the end cell lysates were subjected to Western blot analysis using an antibody against acetyl-H4. The $\mathrm{x}$-axis indicates the concentrations of HDACIs on logarithmic scale; the $\mathrm{y}$-axis indicates the intensity of the bands corresponding to acetyl-H4. Actin blot was included to show equal protein loading for all the samples. The results were representative of three independent experiments \pm SE. 


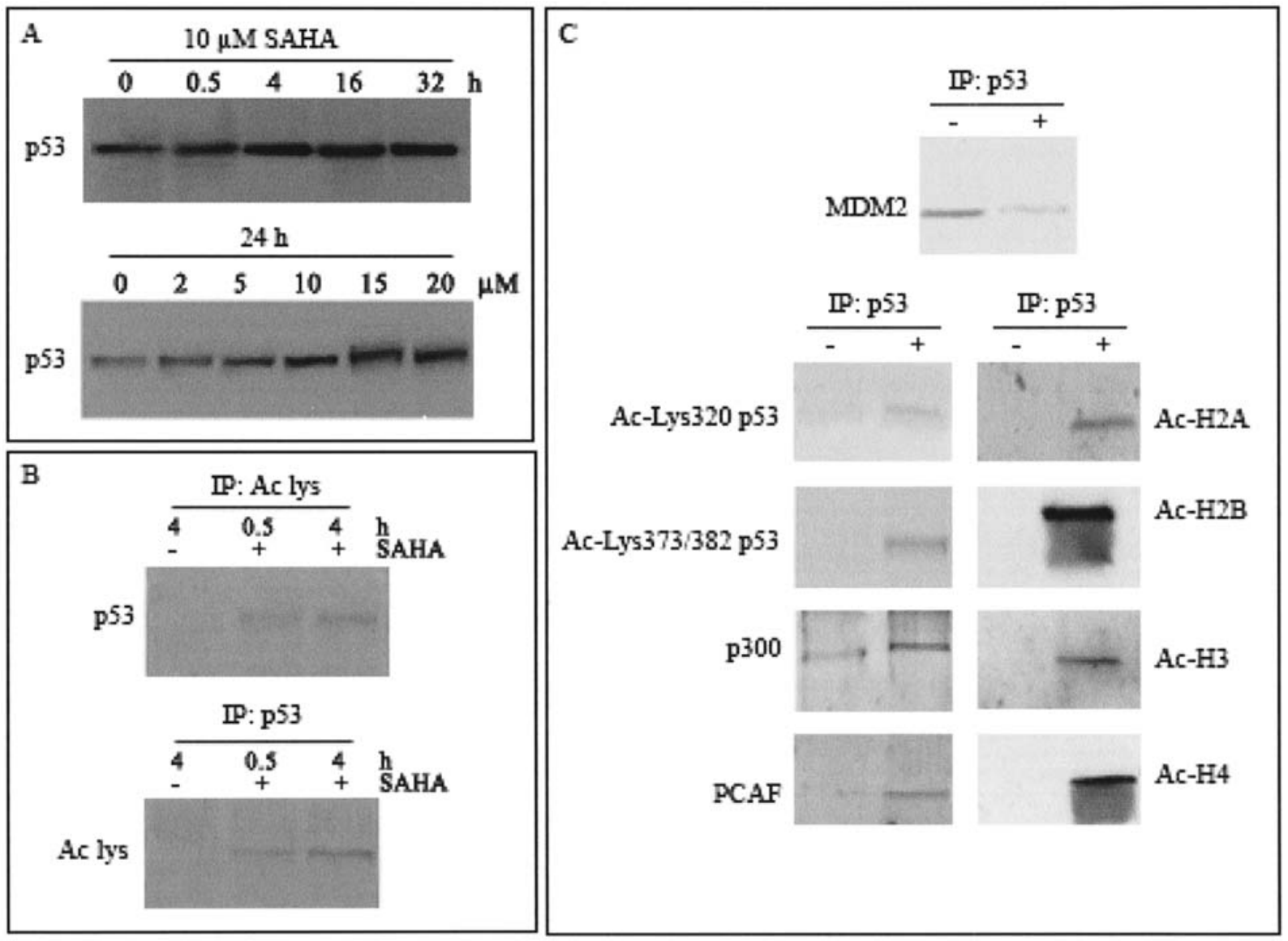

Figure 4. The effect induced by SAHA on p53 and its acetylation. The interaction of acetylated p53 with acetylated histones and coactivators. (A) HepG2 cells were treated with $10 \mu \mathrm{M}$ SAHA for various times or with various doses of SAHA for $24 \mathrm{~h}$. At the end the level of p53 was ascertained by means of Western blot using p53 antibody DO1. (B and C) HepG2 cells were treated for various times (B) or for $4 \mathrm{~h}(\mathrm{C})$ with $10 \mu \mathrm{M}$ SAHA. At the end nuclear extracts were prepared and immunoprecipitated with antibodies against p53 (B and C) or acetyl-lysine (B). The presence of p53, acetyl-lysine containing proteins, AcLys 320 p53, Ac-Lys 373 p53, MDM2, p300, PCAF, acetyl-H2A, acetyl-H2B, acetyl-H3 or acetyl-H4 was revealed using specific antibodies. Actin blot was included to show equal protein loading for all the samples.

coactivators. As shown in Fig. 4A, treatment with $10 \mu \mathrm{M}$ SAHA induced an increase in the level of p53. The effect, which was time- and dose-dependent, was observed already at 30 min of treatment and gradually increased with the time of exposure to the drug, reaching at $32 \mathrm{~h}$ a value that was 2 -fold higher than that of untreated cells.

Nuclear fraction, prepared as described in Materials and methods, was employed for immunoprecipitation procedures. For all these experiments HepG2 cells were treated with $10 \mu \mathrm{M}$ SAHA for $4 \mathrm{~h}$. We first performed immunoprecipitation using an antibody against acetyl-lysine and the immunoprecipitates were then submitted to Western blot analysis. Detection performed by means of the p53 antibody DO1 revealed the presence in treated cells of a significant level of p53 (Fig. 4B). Similar results were also obtained when nuclear extracts of SAHA-treated cells were immunoprecipitated with the p53 antibody DO1 and then subjected to Western blot analysis using for the detection an antibody against acetyl-lysine (Fig. 4B). Moreover specific antibodies revealed that in SAHA-treated cells p53 can be acetylated both at lysine 320 and lysines 373/382 (Fig. 4C). These results taken together clearly indicate that SAHA induced acetylation of p53.

Immunoprecipitates obtained using the p53 antibody DO1 were also employed to study possible interactions of p53 with other proteins (Fig. 4C). Western blot detected with specific antibodies indicated the presence in the immunoprecipitates of p300/CBP. Also the other acetyltransferase PCAF was detected, but at a low level. It is interesting to note that SAHA treatment induced the presence in the immunoprecipitates of remarkable levels of acetylated histones $\mathrm{H} 2 \mathrm{~A}, \mathrm{H} 2 \mathrm{~B}$ and $\mathrm{H} 4$, while the amount of the acetylated form of histone $\mathrm{H} 3$ seemed to be less abundant (Fig. 4C). These results suggested that SAHA treatment favoured the interaction of p53 with the acetylated forms of histones and coactivators.

It has been reported that MDM2 interacts with p53, inhibiting its transcriptional function (20). Thus, to rule out the possibility that in HepG2 cells p53 can interact with MDM2, immunoprecipitation experiments were carried out using the p53 antibody DO1. As seen in Fig. 4C, in untreated HepG2 cells MDM2 protein was immunoprecipitated with the 553 antibody DO1, indicating that under these conditions p53 binds to MDM2. When the cells were treated for $4 \mathrm{~h}$ with $10 \mu \mathrm{M}$ SAHA, p53 failed to co-immunoprecipitate MDM2, suggesting that SAHA treatment weakened the interaction of $\mathrm{p} 53$ with MDM2.

The influence of pifithrin- $\alpha$ on the effects exerted by SAHA on p53 and its acetylated form and the induction of apoptosis. 

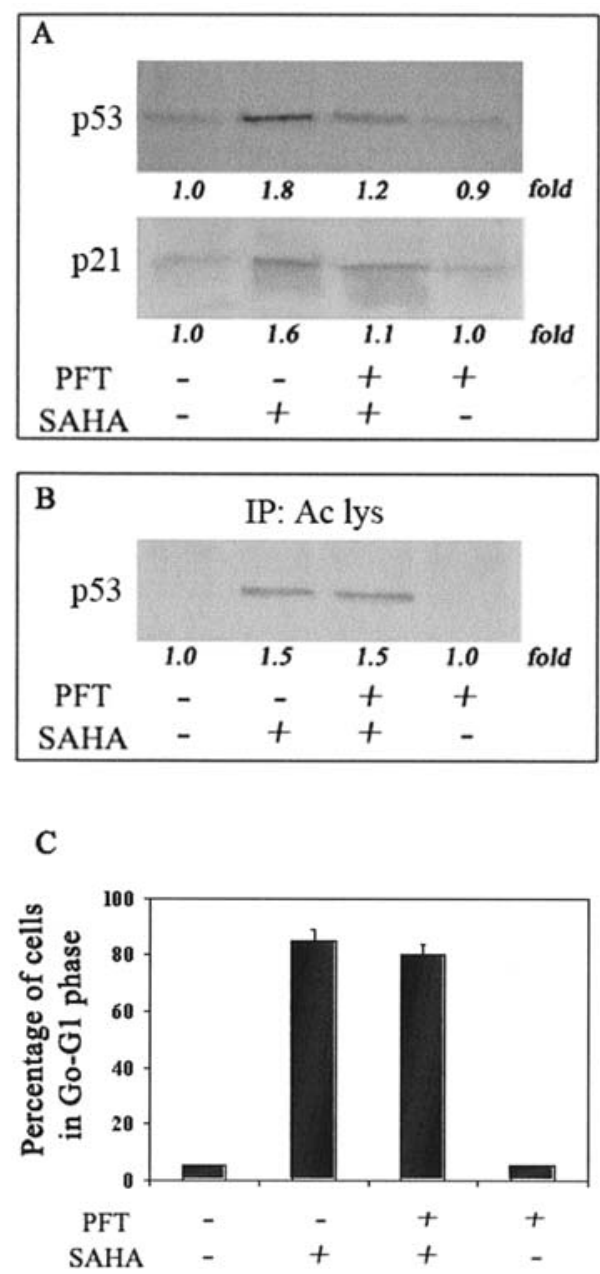

Figure 5. The influence of pifithrin- $\alpha$ on the effects exerted by SAHA on the levels of p53 and acetylated p53 in comparison with the induction of apoptosis. HepG2 cells were treated for $10 \mathrm{~h}$ without or with $20 \mu \mathrm{M}$ PFT- $\alpha$. Then SAHA was added and the incubation was protracted for other $24 \mathrm{~h}$. Western blot analysis of p53 was performed using (A) total extracts of HepG2 cells or (B) immunoprecipitates obtained with acetylated-lysine antibody from nuclear extracts of HepG2 cells. (C) The influence of PFT- $\alpha$ on the apoptotic effect exerted by SAHA on HepG2 cells evaluated by flow cytometric analysis.

Pifithrin- $\alpha$ (PFT- $\alpha)$ has been shown to inhibit transcriptional activity of p53 (21). We performed some experiments in order to ascertain the influence of PFT- $\alpha$ on the effects exerted by SAHA both on p53 and p21 levels and on apoptosis. In these experiments HepG2 cells were treated for $10 \mathrm{~h}$ without or with $20 \mu \mathrm{M}$ PFT- $\alpha$ then SAHA was added and the incubation was protracted for other $24 \mathrm{~h}$. Results shown in Fig. 5A demonstrated that treatment with $10 \mu \mathrm{M}$ SAHA increased the levels of p53 and p21, while the addition of PFT- $\alpha$ significantly reduced both these effects.

Moreover, in order to evaluate the effect of PFT- $\alpha$ on p53 acetylation, we performed immunoprecipitation with the antibody against acetyl-lysine, followed by Western blot with p53 antibody. As shown in Fig. 5B, PFT- $\alpha$ did not modify the effect of SAHA on the level of acetylated p53.

As shown in Fig. 5C, PFT- $\alpha$ did not exert any apoptotic effect on HepG2 cells and moreover did not modify the apoptotic effect induced by SAHA.
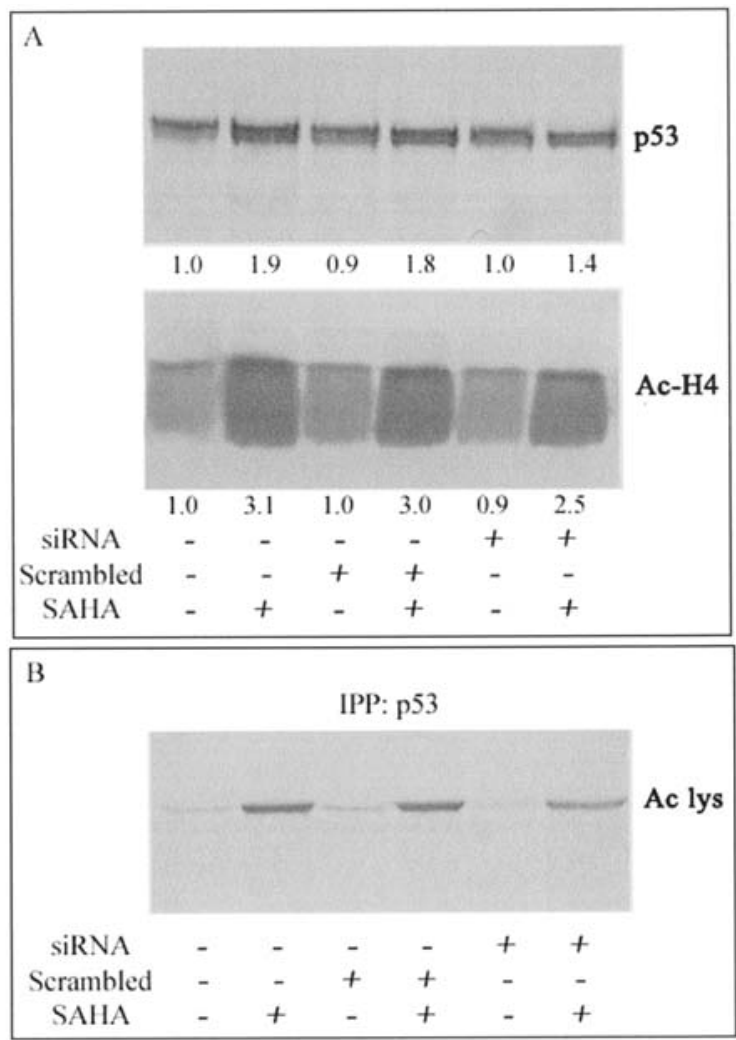

C

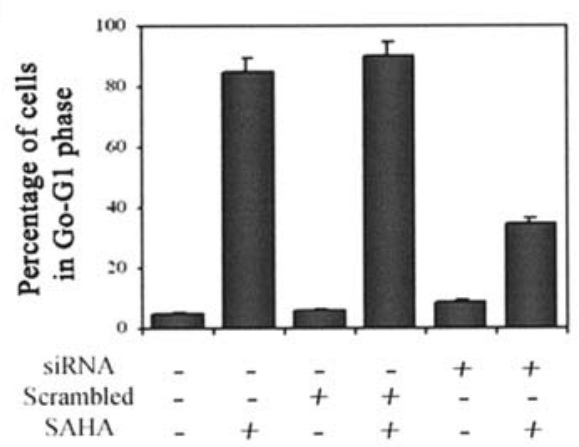

Figure 6. Effects of p53 siRNA on HepG2 cells. HepG2 cells were transfected for $24 \mathrm{~h}$ with 80 pmoles of p53 siRNA or non-silencing scrambled siRNA as described in Materials and methods. Cells were then incubated for other $24 \mathrm{~h}$ with $10 \mu \mathrm{M}$ SAHA. At the end (A) total extracts were subjected to Western blot analysis using antibodies against p53 or acetyl-H4 or (B) nuclear extracts were prepared, immunoprecipitated with p53 antibody DO1 and analysed for Western blot using acetyl-lysine antibody. (C) The influence of p53 siRNA on the apoptotic effect exerted by SAHA was evaluated by flow cytometric analysis.

p53 siRNA lowered the effect of SAHA on apoptosis. To further investigate the role of $\mathrm{p} 53$ and its acetylated form on apoptosis induced by SAHA, HepG2 cells were transfected with 80 pmol siRNA directed against p53 or with scrambled siRNA. Twenty-four hours after transfection, HepG2 cells were treated with $10 \mu \mathrm{M}$ SAHA for $24 \mathrm{~h}$ and cell lysates were analysed by Western blot to evaluate $\mathrm{p} 53$. As reported in Fig. 6A, a remarkable decrease in total p53 was found in cells transfected with p53 siRNA, while this effect was not observed in HepG2 cells transfected with scrambled sequence. Moreover, in order to evaluate the effect of p53 
siRNA on p53 acetylation, we performed immunoprecipitation with the antibody against p53, followed by Western blot with acetyl-lysine antibody. As shown in Fig. 6B, also a remarkable decrease in the level of acetylated p53 was observed in cells transfected with p53 siRNA.

Western blot analysis demonstrated that treatment with siRNA against p53 also decreased the level of acetylated form of histone $\mathrm{H} 4$ in SAHA-treated cells, but the effect was more modest than that found for p53 (Fig. 6A).

Accordingly with a crucial role of $\mathrm{p} 53$ in inducing apoptotic effects, transfection with p53 siRNA markedly reduced the apoptotic effect induced in HepG2 cells by treatment with SAHA (Fig. 6C).

\section{Discussion}

It is well known that p53 is a transcription factor involved in the transactivation of genes which are responsible for different processes, such as cell cycle arrest, DNA repair and apoptosis (22). p53 exhibits three different domains (23): the transactivation domain, the tetramerization domain and the sequence-specific DNA binding domain. In addition, p53 contains a C-terminal basic domain which shows distinct DNA-binding properties and that can be acetylated in response to DNA damage $(24,25)$. These acetylation events enhance p53 DNA-binding activity and lead to activation of its ability to induce cell cycle arrest or apoptosis (26). Two distinct classes of co-activators/acetyltransferases are involved in lysine acetylation of p53 (27). p300/CBP acetylates lysines located in the last carboxy-terminal portion of p53, in particular Lys-382 and, to a less extent, Lys-373 while p300/ CBP-associated factor (PCAF) intervenes in the acetylation of Lys-320, located near the oligomerization domain of the protein. Both p300/CBP and PCAF are considered as histone acetyltransferases (HATs) since they are implicated in acetylation of the amino terminal domains of histone proteins included in the nucleosomes with consequent relaxation of chromatin structure (28). In addition, recently, it has been demonstrated that acetylation of Lys 120, located in the DNA binding domain of p53, occurs rapidly after DNA damage (25). Such an event, which is catalysed by the MYST family acetyltranferases hMOF and TIP60 (29), seems to be correlated with the transcriptional activation of pro-apoptotic target genes, such as Bax and PUMA (25).

In unstressed cells p53 protein is present at a very low level. This fact is mostly caused by p53 degradation assured by interaction with MDM2 rather than a low rate of mRNA translation (30). MDM2 is an E3 ubiquitin ligase which causes ubiquitination of p53 with the consequent degradation by the proteasome. Phosphorylation at Ser 15, Thr 18 and Ser 20 stimulates the recruitment of acetyltransferases, such as p300 and PCAF, that can thus promote p53 C-terminal acetylation (31). Such an event prevents ubiquitination and degradation, thereby favouring p53 stabilization.

Our results show that histone deacetylase inhibitors (HDACIs) induced apoptosis in hepatoma cells in a doseand time-dependent manner. Among the compounds tested TSA, ITF2357 and SAHA were very effective in apoptosis induction in HepG2 cells, while valproic acid and sodium butyrate showed a much lower activity. However, in all cases apoptosis was observed after a lag phase of about 12-16 h.

It is interesting to note that HDACIs increased the level of p53 protein and rapidly induced acetylation of p53 and histones. These effects were observed at doses of HDACIs which were effective in inducing apoptosis.

In this connection it is important to note that pifithrin- $\alpha$, a compound capable of suppressing p53-mediated transactivation (21), induced a remarkable decrease in the level of p53 and its transcriptional target p21, most likely by favouring p53 degradation, but did not modify the effect of SAHA on the level of acetylated p53. Since PFT- $\alpha$ also did not modify the effect exerted on apoptosis by SAHA, we suggest that apoptosis can be correlated with acetylated p53 rather than with total p53.

Another interesting aspect of our results was the observation that treatment with SAHA produced complexes containing acetylated p53 together with acetylated forms of histones and co-activators. Concomitantly SAHA induced the release of MDM2 from p53, most likely as a consequence of p53 acetylation. These findings suggest that acetylation of p53 can allow its stabilization in the nucleus thus favouring the production of nuclear protein complexes. Moreover, it seems possible that a direct relationship exists between the acetylated forms of p53 and histones.

This conclusion was suggested by the observation that silencing of p53 induced a marked decrease in the level of acetylated p53 and a concomitant decrease in the level of acetylated histones, although to a minor extent. Silencing of p53 also markedly reduced the apoptotic action exerted by SAHA in HepG2 cells.

To explain these results, we suggest that acetylated p53 plays a crucial role in the production of nuclear complexes by recruiting histone acetyltransferases (HATs) that are involved in mediating histone acetylation. Moreover, it seems possible that bromodomains of HATs can interact with acetylated histones favouring the production of large complexes which are involved in the induction of apoptosis. This conclusion is in accordance with the results of Barlev et al (27) showing that acetylation of p53 and its association with coactivators is required for the physiologic function of p53 in cell cycle arrest following DNA damage (27) and with the finding of Avantaggiati et al which showed that p53 forms a specific protein complex with p300 (32).

However, it seems possible that acetylation of p53 can occur at levels different from lys 373 and 320 and that acetylation of the protein requires the involvement of acetyltransferases different from p300 and PCAF, such as acetyltransferases of the MYST family and in particular hMOF and TIP60. We are undertaking further studies to individuate whether other activities can be involved in the acetylation induced by HDACIs.

It is well known that post-translational covalent modifications of the histones within the nucleosomes are responsible for a complex epigenetic program that controls gene expression in eukaryotes. Such modifications of histones occur on the amino-terminal tails and include acetylation, phosphorylation, ubiquitination, methylation and sumoylation. Histone modifications form a 'histone code' $(4,33)$ that is involved in the recruitment of transcription factors of other regulatory 
proteins containing specific interacting domains: bromodomains $(34,35)$ and chromodomains $(36,37)$. Therefore, post-translational modifications of histones play an important role in the coordination of gene expression correlated with cell proliferation or apoptosis.

Acetylation of histone tails, occurring for the four histones at the $\varepsilon$-amino groups of lysine residues, can be considered as the most studied modification of the histone code. In vivo lysine acetylation is governed by a dynamic equilibrium between acetyltransferases and deacetylases. In the case of SAHA-induced apoptosis, the activity of HDACs is inhibited while acetyltranferases were immunoprecipitated together with p53 and histones, and they are most likely responsible for acetylation of both p53 and histones.

We propose that p53 and histones cooperate in the induction of apoptosis and suggest that the histone code together with post-translational modifications of p53 can be responsible for the regulation of pro-apoptotic gene expression. On the other hand, it is not possible to exclude that in other cells and circumstances acetylation of histones can play a role independent of p53 in the mechanism of HDACIs-induced apoptosis.

Many lines of evidence suggest that histone hypo-acetylation and overexpression of HDACs are involved in tumorigenesis of various human malignancies (38) and that histone hypo-acetylation induces repression of tumor suppressor gene expression (4).

Our results, showing that HDACIs rapidly stimulate acetylation of histones, suggest that these compounds can depress gene expression increasing the susceptibility of tumor cells to apoptosis. Therefore, HDACIs can be useful in patients at high risk of cancers or with precancerous conditions. Finally the addition of HDACIs into clinical trials can contribute to reducing the active doses of many apoptotic agents, thus diminishing their toxic effects.

\section{Acknowledgments}

We thank Dr Paolo Mascagni and Italfarmaco for kindly providing SAHA and ITF2357 employed for this research.

\section{References}

1. Kristeleit R, Stimson L, Workman P and Aherne W: Histone modification enzymes: novel targets for cancer drugs. Expert Opin Emerg Drugs 9: 135-154, 2004.

2. Peart MJ, Smyth GK, van Laar RK, et al: Identification and functional significance of genes regulated by structurally different histone deacetylase inhibitors. Proc Natl Acad Sci USA 102: 3697-3702, 2005.

3. Gray SG and Ekstrom TJ: The human histone deacetylase family. Exp Cell Res 262: 75-83, 2001

4. Santos-Rosa $\mathrm{H}$ and Caldas C: Chromatin modifier enzymes, the histone code and cancer. Eur J Cancer 41: 2381-2402, 2005.

5. Liu T, Kuljaca S, Tee A and Marshall GM: Histone deacetylase inhibitors: multifunctional anticancer agents. Cancer Treat Rev 32: 157-165, 2006.

6. Newmark HL and Young CW: Butyrate and phenylacetate as differentiating agents: practical problems and opportunities. J Cell Biochem Suppl 22: 247-253, 1995.

7. Bouchain G and Delorme D: Novel hydroxamate and anilide derivatives as potent histone deacetylase inhibitors: synthesis and antiproliferative evaluation. Curr Med Chem 10: 2359-2372, 2003.
8. Marks PA, Richon VM and Rifkind RA: Histone deacetylase inhibitors: inducers of differentiation or apoptosis of transformed cells. J Natl Cancer Inst 92: 1210-1216, 2000.

9. Somech R, Izraeli S and J Simon A: Histone deacetylase inhibitors - a new tool to treat cancer. Cancer Treat Rev 30: 461-472, 2004.

10. Mitsiades CS, Mitsiades NS, McMullan CJ, et al: Transcriptional signature of histone deacetylase inhibition in multiple myeloma. Biological and clinical implications. Proc Natl Acad Sci USA 101: 540-545, 2004

11. Shankar S, Singh TR, Fandy TE, Luetrakul T, Ross DD and Srivastava RK: Interactive effects of histone deacetylase inhibitors and TRAIL on apoptosis in human leukemia cells: involvement of both death receptor and mitochondrial pathways. Int J Mol Med 16: 1125-1138, 2005.

12. Bali P, Pranpat M, Swaby R, et al: Activity of suberoylanilide hydroxamic acid against human breast cancer cells with amplification of Her-2. Clin Cancer Res 11: 6382-6389, 2005.

13. Mitsiades CS, Poulaki V, McMullan C, et al: Novel histone deacetylase inhibitors in the treatment of thyroid cancer. Clin Cancer Res 11: 3958-3965, 2005.

14. Kelly WK and Marks PA: Drug insight: histone deacetylase inhibitors - development of the new targeted anticancer agent suberoylanilide hydroxamic acid. Nat Clin Pract Oncol 3: 150-157, 2005.

15. Kelly WK, O'Connor OA, Krug LM, et al: Phase I study of an oral histone deacetylase inhibitor, suberoylanilide hydroxamic acid, in patients with advanced cancer. J Clin Oncol 23: 3923-3931, 2005.

16. Emanuele S, Vento R, Tesoriere G, et al: SAHA induces apoptosis in hepatoma cells and synergistically interacts with the proteasome inhibitor Bortezomib. Apoptosis 12: 1327-1338, 2007.

17. Lauricella M, Vento R, Tesoriere G, et al: JNK and AP-1 mediate apoptosis induced by bortezomib in HepG2 cells via FasL/ caspase-8 and mitochondria-dependent pathways. Apoptosis 11: 607-625, 2006.

18. Emanuele S, Vento R, Tesoriere G, et al: Apoptosis induced in hepatoblastoma HepG2 cells by the proteasome inhibitor MG132 is associated with hydrogen peroxide production, expression of Bcl-XS and activation of caspase-3. Int J Oncol 21: 857-865, 2002.

19. Lowry OH, Rosebrough NJ, Farr AL and Randall RJ: Protein measurement with the Folin phenol reagent. J Biol Chem 193: 265-275, 1951.

20. Ma J, Martin JD, Zhang H, et al: A second p53 binding site in the central domain of Mdm2 is essential for p53 ubiquitination. Biochemistry 45: 9238-9245, 2006.

21. Rocha S, Campbell KJ, Roche KC and Perkins ND: The p53inhibitor pifithrin-alpha inhibits firefly luciferase activity in vivo and in vitro. BMC Mol Biol 4: 9, 2003.

22. Hofseth LJ, Hussain SP and Harris CC: p53: 25 years after its discovery. Trends Pharmacol Sci 25: 177-181, 2004.

23. Harms KL and Chen X: The functional domains in p53 family proteins exhibit both common and distinct properties. Cell Death Differ 13: 890-897, 2006.

24. Friedler A, Veprintsev DB, Freund SM, von Glos KI and Fersht AR: Modulation of binding of DNA to the C-terminal domain of p53 by acetylation. Structure 13: 629-636, 2005.

25. Sykes SM, Mellert HS, Holbert MA, Li K, Marmorstein R, Lane WS and McMahon SB: Acetylation of the p53 DNAbinding domain regulates apoptosis induction. Mol Cell 24: 841-851, 2006.

26. Smith L and La Thangue NB: Signalling DNA damage by regulating p53 co-factor activity. Cell Cycle 4: 30-32, 2005.

27. Barlev NA, Liu L, Chehab NH, Mansfield K, Harris KG, Halazonetis TD and Berger SL: Acetylation of p53 activates transcription through recruitment of coactivators/histone acetyltransferases. Mol Cell 8: 1243-1254, 2001.

28. Curtis AM, Seo SB, Westgate EJ, et al: Histone acetyltransferasedependent chromatin remodeling and the vascular clock. J Biol Chem 279: 7091-7097, 2004.

29. Sterner DE and Berger SL: Acetylation of histones and transcription-related factors. Microbiol Mol Biol Rev 64: 435-459, 2000.

30. Tang J, Qu LK, Zhang J, et al: Critical role for Daxx in regulating Mdm2. Nat Cell Biol 8: 855-862, 2006. 
31. Knights CD, Catania J, Di Giovanni S, et al: Distinct p53 acetylation cassettes differentially influence gene-expression patterns and cell fate. J Cell Biol 173: 533-544, 2006.

32. Avantaggiati ML, Ogryzko V, Gardner K, Giordano A, Levine AS and Kelly K: Recruitment of p300/CBP in p53-dependent signal pathways. Cell 89: 1175-1184, 1997.

33. Villar-Garea A and Imhof A: The analysis of histone modifications. Biochim Biophys Acta 1764: 1932-1939, 2006.

34. Mujtaba S, He Y, Zeng L, et al: Structural mechanism of the bromodomain of the coactivator CBP in p53 transcriptional activation. Mol Cell 13: 251-263, 2004.
35. Winston F and Allis CD: The bromodomain: a chromatintargeting module? Nat Struct Biol 6: 601, 1999.

36. De la Cruz X, Lois S, Sanchez-Molina S and Martinez-Balbas MA: Do protein motifs read the histone code? Bioessays 27: 164-175, 2005.

37. Brehm A, Tufteland KR, Aasland R and Becker PB: The many colours of chromodomains. Bioessays 26: 133-140, 2004.

38. Cairns BR: Emerging roles for chromatin remodelling in cancer biology. Trends Cell Biol 11: 15-21, 2001. 